\title{
Habilidades sociais educativas de mães separadas e sua relação com o comportamento de pré- escolares
}

\author{
Ana Carolina Villares Barral Villas Boas' - Universidade de Brasilia, Brasilia, Brasil \\ Alessandra Turini Bolsoni-Silva - Universidade Estadual Paulista, Bauru, Brasil
}

\begin{abstract}
Resumo
Este estudo teve por objetivo: (a) caracterizar uma amostra de mães e filhos, em famílias separadas, quanto às suas habilidades sociais; (b) investigar relações entre as habilidades sociais educativas das mães e o comportamento das crianças; e (c) comparar grupo clínico e não-clínico quanto ao repertório comportamental. Participaram da pesquisa 43 mães de crianças de ambos os sexos, com idade entre quatro e seis anos. As participantes responderam a um Questionário Sociodemográfico, Roteiro de Entrevista de Habilidades Sociais Educativas Parentais, Child Behavior Checklist - CBCL, Questionário de Respostas Socialmente Habilidosas e Escala Infantil A2 de Rutter. Para análise de dados, empregaram-se os testes Spearman e Mann-Whitney. Os resultados mostraram que habilidades sociais educativas das mães estiveram relacionadas a habilidades sociais das crianças, enquanto práticas negativas foram relacionadas a problemas de comportamento. Discutem-se como as habilidades sociais maternas podem influenciar o comportamento dos filhos em famílias separadas.

Palavras-chave: Habilidades sociais educativas parentais, Separação conjugal, Comportamento infantil.
\end{abstract}

\section{Educational social skills of separated mothers and their relation to preschoolers' behavior}

\begin{abstract}
This study aimed to: (a) characterize a sample of mothers and children in separated families with regard to their social skills; (b) investigate the relation between mothers' social skills and children's behavior; and (c) compare clinical and nonclinical sample as to the behavioral repertory. Forty three mothers of children of both sexes, aged between four and six years participated in the study. Mothers answered a Sociodemographic Questionnaire, an Interview about Parental Educational Social Skills, the Child Behavior Checklist - CBCL, a Questionnaire of Social Skills Responses, and Rutter's A2 Scale. For data analysis, Spearman and Mann-Whitney tests were used. Results showed that mothers' educational social skills were related to social skills of children, while negative practices were associated with behavioral problems. We discuss how maternal social skills may influence children's behavior in separated families.

Keywords: Parental educational social skills, Marriage separation, Child behavior.
\end{abstract}

A relação entre pais e filhos vem sendo bastante discutida na literatura, e muitos pesquisadores sugerem uma relação entre o modo de educar dos pais e a construção das habilidades sociais e da competência social dos filhos (Bolsoni-Silva, 2003; Gomide, 2001; Pacheco, Teixeira \& Gomes, 1999; Salvo, Silvares \& Toni, 2005). Como afirmam Del Prette e Del Prette (2002a), as habilidades sociais da criança são adquiridas, geralmente, por meio da mediação dos pais e, sendo assim, o contexto familiar, o envolvimento e o desempenho parental são extremamente importantes para o estabelecimento de relações educativas que de fato promovam o desenvolvimento social dos filhos (Cia, Pereira, Del Prette \& Del Prette, 2006). Entretanto, não se deve deixar de mencionar que a criança exerce um papel importante no seu processo de socialização e, portanto, também influencia o relacionamento com seus pais (Biasoli-Alves \& Sigolo, 1992).

\footnotetext{
${ }^{1}$ Endereço para correspondência:

Departamento de Psicologia, Faculdade de Ciências - Unesp

Av. Eng. Luiz Edmundo Carrijo Coube, no 14-01 - Vargem Limpa 17033-360 - Bauru-SP

E-mail: villares_ana@yahoo.com.br
}

Del Prette e Del Prette (2002a) definem habilidades sociais educativas como um conjunto de habilidades destinadas intencionalmente a promover a aprendizagem ou o desenvolvimento do outro, seja em situação formal ou informal. No contexto familiar, para estudar a relação entre pais e filhos, Bolsoni-Silva (2003) empregou o termo habilidades sociais educativas parentais, que se refere ao conjunto de habilidades sociais aplicadas especificamente à educação dos filhos, tais como as habilidades envolvidas no estabelecimento de limites, na comunicação e na expressão de sentimentos e opiniões.

Diversas pesquisas têm reportado as implicações das práticas educativas e das habilidades sociais educativas parentais para o comportamento infantil, seja contribuindo para o desenvolvimento de habilidades sociais, seja correlacionando determinadas práticas a problemas de comportamento (Alvarenga \& Piccinini, 2001; Bolsoni-Silva, 2003; Bolsoni-Silva \& Marturano, 2002; Brenner \& Fox, 1998; Kaiser \& Hester, 1997; Kerr, Lopez, Olson \& Sameroff, 2004; Pacheco \& cols., 1999; Patterson, Reid \& Dishion, 2002; Salvo \& cols., 2005). Algumas dessas pesquisas (Brenner \& Fox, 1998; Kaiser \& Hester, 1997; Kerr \& cols., 2004; Patterson \& cols., 2002) demonstram que práticas educativas consideradas inadequadas, como o 
uso frequente da punição, podem levar a criança a se comportar de modo coercitivo diante de pares e de professores, principalmente os meninos, visando à obtenção de reforçadores positivos e negativos (Goldiamond, 1974/2002).

Em famílias separadas, estudos mostram que as práticas educativas dos pais também estão associadas ao comportamento dos filhos (Wood, Repetti \& Roesch, 2004) e podem funcionar como mediadoras dos efeitos da separação sobre problemas de comportamento das crianças (Pett, Wampold, Turner \& Vaughan-Cole, 1999). Wolchik, Wilcox, Tein e Sandler (2000), por exemplo, observaram que práticas educativas maternas relacionadas à aceitação e à consistência na disciplina minimizaram os efeitos negativos da separação para os filhos, especificamente, o surgimento de problemas de comportamento. Os autores consideraram que, ao manter diálogos com os filhos, as mães poderiam ajudá-los a lidar com dificuldades advindas da transição na estrutura familiar, por exemplo, com o medo do abandono em relação às figuras parentais. Já a consistência na disciplina possibilitaria à criança certa previsibilidade sobre os eventos, o que favoreceria maior sensação de controle sobre seu ambiente. $\mathrm{Na}$ mesma direção, estudos de intervenção com famílias separadas têm mostrado que a promoção de habilidades de comunicação, de consistência na disciplina e de interações positivas entre pais e filhos pode contribuir para a redução de problemas de comportamento e proporcionar relações mais satisfatórias (Almeida, Moraes, Perez, Garcia \& Pellizari, 2000; Tein, Sandler, MacKinnon \& Wolchik, 2004).

Portanto, pesquisadores têm reportado que crianças que mantêm um bom relacionamento com suas mães apresentam menos problemas, o que sugere que mães com mais habilidades sociais educativas podem prevenir o surgimento e a manutenção de problemas de comportamento e favorecer o desenvolvimento de um repertório socialmente habilidoso (Wolchik \& cols., 2000; Wood \& cols., 2004). Por outro lado, aqueles pais que apresentam importantes déficits em habilidades sociais educativas parentais, como em manter conversação e demonstrar afeto ou, ainda, fazem uso de estratégias coercitivas para disciplinar os filhos e apresentam práticas inconsistentes, podem colocar a criança em situação de risco para a emergência de problemas de comportamento (Wolchik \& cols., 2000). Como afirmam Hetherington e Stanley-Hagan (1999), filhos de pais separados podem se tornar socialmente competentes quando o pai/mãe que detém a guarda da criança for capaz de prover um ambiente de cuidado positivo, o que significa que muito do que a criança aprende está relacionado às habilidades dos pais diante da sua educação.

Embora estudos apontem para os efeitos deletérios da separação conjugal (Capaldi \& Patterson, 1991; Cherlin \& cols., 1991; Lansford \& cols., 2006), pesquisadores têm constatado que a maioria das crianças não apresenta problemas emocionais ou comportamentais em decorrência da separação parental (Amato, 2000; Amato \& Keith, 1991; Lansford, 2009). $O$ fato de nem todas as crianças apresentarem problemas tem levado pesquisadores a avaliar quais fatores estão contribuindo para essa variabilidade (Amato, 2000; Lansford, 2009; Morrison \& Cherlin, 1995; Pett \& cols., 1999; Pruett, Williams \& Insabella, 2003; Whiteside \& Becker, 2000; Wolchik \& cols., 2000). Na perspectiva de Matos (1983), pesquisas sobre o desenvolvimento infantil devem analisar características mais proximais do ambiente, como as interações que nele ocorrem e que podem ajudar a compreender quais fatores são responsáveis pela variabilidade observada no comportamento das crianças.

Investigar as habilidades sociais educativas parentais pode oferecer informações relevantes sobre o contexto de desenvolvimento das crianças e ajudar a compreender a relação entre as habilidades dos pais e o comportamento dos filhos. Dessa forma, este estudo teve por objetivo descrever as habilidades sociais educativas de mães separadas e suas possíveis relações com o comportamento dos filhos, considerando tanto o comportamento socialmente habilidoso quanto problemas de comportamento da criança. Para tanto, buscou-se: (a) caracterizar a amostra no que se refere às habilidades sociais de mães e filhos; (b) correlacionar o comportamento de mães e filhos com base em diferentes instrumentos; e (c) comparar grupos caracterizados como clínico e não-clínico quanto ao repertório de mães e de filhos.

\section{Método}

\section{Participantes}

Participaram da pesquisa 43 mães de crianças de ambos os sexos (26 meninos e 17 meninas), com idade entre quatro e seis anos $(M=4,8 ; \mathrm{DP}=0,8)$ e, em média, 2,7 anos na época da separação dos pais. As crianças estavam matriculadas em nove Escolas Municipais de Educação Infantil (EMEIs) de uma cidade do interior do estado de São Paulo.

A média de idade das mães era de 30,5 anos $(\mathrm{DP}=6,5)$ e 2,1 filhos $(\mathrm{DP}=1,0)$, sendo que as participantes estavam separadas do ex-companheiro há, em média, 2,5 anos $(\mathrm{DP}=1,4)$. Quanto ao grau de escolaridade, $26 \%$ das mães tinham o ensino 
fundamental incompleto e a mesma proporção, o ensino fundamental completo. Trinta e nove por cento tinham o ensino médio completo e $9 \%$ haviam concluído o nível superior. A renda materna situava-se entre $\mathrm{R} \$ 100,00$ e $\mathrm{R} \$ 500,00$ para $44,2 \%$ da amostra, enquanto $16,2 \%$ recebiam entre $\mathrm{R} \$ 600,00$ e $\mathrm{R} \$$ $1.000,00$, e $14 \%$ tinham renda superior a $\mathrm{R} \$ 1.100,00$. Outros $25,6 \%$ das participantes não tinham renda própria. Conforme relato das participantes, $67 \%$ delas recebiam contribuição financeira do ex-companheiro.

\section{Instrumentos}

Roteiro de Entrevista de Habilidades Sociais Educativas Parentais - RE-HSE-P. O roteiro, adaptado de Bolsoni-Silva (2009), é composto por conjuntos de questões que investigam a frequência e a qualificação de habilidades sociais maternas e infantis, além de práticas negativas de educação, problemas de comportamento e variáveis contextuais, num total de 70 categorias. A mãe é questionada, por exemplo, se estabelece limites ao seu filho e, ao responder que sim, investiga-se a frequência do comportamento. $\mathrm{Na}$ sequência, responde à pergunta "Como você estabelece limites para o seu filho?" e, ainda nesse bloco de informações, pergunta-se como se sente e como o filho se comporta nessas horas. Bolsoni-Silva (2009) encontrou satisfatória consistência interna (alfa de Chronbach $=0,83$ ), com dois fatores: um positivo (hse$\mathrm{p}$, habilidades sociais infantis, contexto, total positivo) e um negativo (práticas negativas, problemas de comportamento e total negativo) de interações sociais. O instrumento também discriminou população clínica e não clínica para habilidades sociais educativas parentais, habilidades sociais dos filhos e escore total de interações sociais positivas. É possível realizar uma análise de forma a organizar os dados em três categorias amplas: Comunicação; Expressão de Sentimento e Enfrentamento; e Estabelecimento de Limites.

CBCL (Child Behavior Checklist - Inventário de Comportamentos da Infância e Adolescência para pré-escolares e escolares (4 a 18 anos). Instrumento desenvolvido por Achenbach e Rescorla (2001), que investiga a frequência de respostas para problemas de comportamento, indicando ou não classificação clínica, portanto diagnóstica, para problemas externalizantes, internalizantes e total. $\mathrm{O}$ instrumento possui validação para a população americana, o que significa que sua classificação, quando utilizada na população brasileira, deve ser feita com cautela. Bordin, Mari e Caeiro (1995) conduziram estudos preliminares de validação do inventário, mas até o momento não estão disponíveis softwares para avaliar os comportamentos baseados em referências nacionais.
Questionário de Respostas Socialmente Habilidosas (QRSH - Pais). Visa avaliar comportamentos socialmente habilidosos de crianças com idade entre 4 e 7 anos. Seus itens podem ser agrupados em Disponibilidade Social e Cooperação, Interação Social Positiva e Expressão de Sentimentos e Enfrentamento (Bolsoni-Silva, Marturano, Figueiredo \& Manfrinato, 2006). Em estudo desenvolvido sobre as propriedades psicométricas do questionário, Bolsoni-Silva, Marturano \& Loureiro (no prelo) verificaram que o instrumento apresenta consistência interna satisfatória $(0,82)$.

Escala Comportamental Infantil A2 de Rutter - ECI A2 (adaptado por Graminha, 1994, 1998). Avalia se a criança possui ou não indicativos de problemas de comportamento, e seus itens podem ser organizados em externalizantes, internalizantes e outros problemas (Bolsoni-Silva, Marturano \& Manfrinato, 2005). Esse instrumento não é considerado para diagnóstico, mas consiste em uma espécie de rastreador para problemas de comportamento.

As participantes responderam, ainda, a um Questionário Sociodemográfico, elaborado para a presente pesquisa. Todos os instrumentos utilizados na coleta de dados foram aplicados oralmente pela pesquisadora, que fez o registro das respostas.

\section{Procedimento de coleta de dados}

Antes da coleta de dados e em conformidade com as normas de ética da Resolução 196/96 do Conselho Nacional de Saúde, o projeto de pesquisa foi submetido à apreciação e aprovado pelo Comitê de Ética em Pesquisa da Faculdade de vinculação do estudo. Após essa etapa, as participantes foram selecionadas por meio da colaboração de professoras das EMEIs, que indicaram, entre seus alunos do Jardim I ao Pré, aqueles que eram filhos de pais separados. As mães das crianças indicadas foram, posteriormente, contatadas e convidadas a participar da pesquisa. As entrevistas com elas foram conduzidas em local reservado nas EMEIs ou na própria residência das famílias. Antes da aplicação dos instrumentos, um Termo de Consentimento Livre e Esclarecido foi empregado para registro da anuência dos envolvidos no estudo.

\section{Procedimentos de análise de dados}

Para a análise dos dados do REHSE-P, inicialmente, atribuiu-se escore às respostas frequentemente (2), algumas vezes (1) e quase nunca/nunca (0), e calculou-se a distribuição das respostas das participantes, bem como o escore total no instrumento. Os dados de frequência (frequência positiva, frequência negativa) e de qualificação (hse-p, 
práticas negativas - negat, problemas de comportamento - probl, habilidades sociais da criança - hs, variáveis de contexto, total negativo e total positivo) foram categorizados e tratados estatisticamente (Teste de Correlação Spearman).

Para análise dos resultados da ECI A2, atribuiu-se escore a cada resposta, de acordo com a instrução de utilização da escala (Graminha, 1998). Os itens foram agrupados em duas categorias referentes a respostas internalizantes e externalizantes, e aqueles que não puderam ser inseridos nessas categorias foram descritos como outras respostas (Bolsoni-Silva \& Marturano, 2002). Calculou-se o escore total do instrumento e o escore parcial de cada categoria. Os dados do QRSH - Pais receberam tratamento idêntico àquele aplicado à ECI A2.

Para verificar a existência de relações entre o comportamento materno e o comportamento das crianças, aplicou-se $\mathrm{o}$ teste não-paramétrico de Spearman no próprio RE-HSE-P (categorias amplas para a HSE-P: Comunicação, Expressão de Sentimentos e Enfrentamento e Estabelecimento de Limites) e entre ele e os outros instrumentos utilizados (ECI A2 e QRSH - Pais). Foram considerados os resultados com significância igual ou inferior a 5\%, expressos na forma de tabelas e figuras.

Para verificar diferenças entre grupos com problemas de comportamento (clínico) versus sem problemas de comportamento (não clínico), foram selecionados 19 participantes que ou tinham problemas de comportamento nas três escalas do CBCL $(n=7)$, ou não apresentavam problemas em nenhuma delas $(n=12)$, cujos dados foram tratados estatisticamente na comparação de grupos (Teste Mann-Whitney).

\section{Resultados}

Quanto ao RE-HSE-P, considerando que foram utilizadas 12 questões do instrumento que avaliavam a frequência das habilidades sociais educativas parentais, a soma dos escores atribuídos às respostas frequentemente, algumas vezes e quase nunca/nunca permitiu uma pontuação que variou de zero a, no máximo, 24 pontos. $O$ escore médio das participantes deste estudo foi de 16 pontos $(\mathrm{DP}=2,65)$, sendo o menor escore sete e o maior, 21 pontos. A caracterização da amostra quanto ao repertório de mães e filhos, conforme RE-HSE-P, é apresentada na Figura 1.
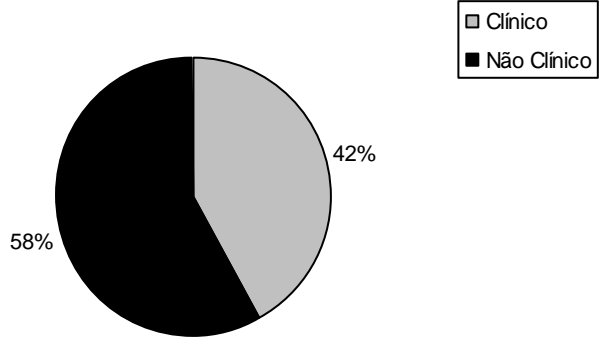

Classificação clínica e não clínica - HSE-P

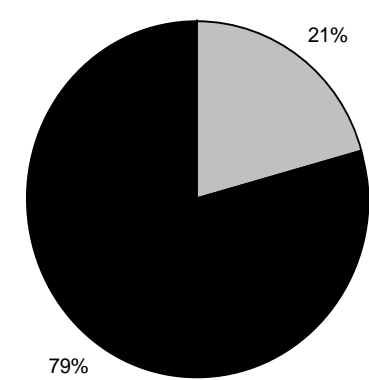

Classificação clínica e não clínica - hs filhos

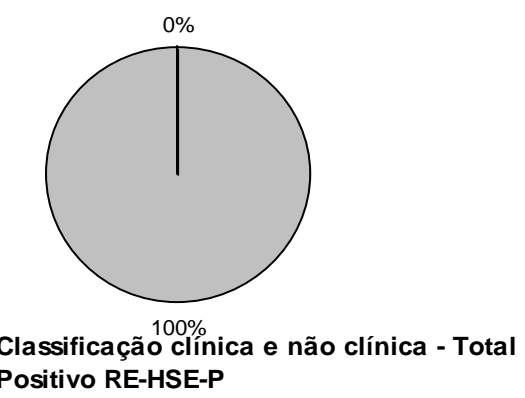

Figura 1 - Porcentagem de mães separadas com classificação clínica e não-clínica, segundo o RE-HSE-P 
De acordo com a Figura 1, nota-se que, considerando o escore total positivo, todos os participantes poderiam ser considerados de grupo clínico, com 58\% das mães compondo um grupo clínico para habilidades sociais educativas e $79 \%$ das crianças no que se refere ao repertório de habilidades sociais. A Tabela 1 apresenta correlações entre os itens do RE-HSE-P, enquanto a Tabela 2 apresenta correlações entre esse instrumento e o QRSH - Pais e a ECI A2, que mensuram habilidades sociais $\mathrm{e}$ indicativos de problemas de comportamento das crianças, respectivamente.

Tabela 1 - Correlações entre itens do RE-HSE-P (Teste Não-Paramétrico de Spearman)

\begin{tabular}{lccccccc}
\hline $\begin{array}{c}\text { Categorias do } \\
\text { RE-HSE-P }\end{array}$ & hse-p & negat & hs & probl & contexto $\begin{array}{c}\text { Total } \\
\text { positivo }\end{array} \begin{array}{c}\text { Total } \\
\text { negativo }\end{array}$ \\
\hline HSE-P & - & & & & & \\
Negat & $-0,210$ & - & & & & \\
HS & 0,284 & 0,052 & - & & & \\
Probl & $-0,198$ & $0,532^{* *}$ & 0,116 & - & & \\
Contexto & $0,334^{*}$ & 0,236 & $0,306^{*}$ & 0,185 & - & & - \\
Total positivo & $0,953^{* *}$ & $-0,237$ & $0,335^{*}$ & $-0,205$ & $0,320^{*}$ & - & \\
Total negativo & $-0,280$ & $0,855^{* *}$ & 0,061 & $0,841^{* *}$ & 0,204 & $-0,311^{*}$ & - \\
${ }^{*} \mathrm{p}<0,05^{* *} \mathrm{p}<0,01$ & & & & & & &
\end{tabular}

$\mathrm{Na}$ Tabela 1, observam-se relações entre habilidades sociais das mães, variáveis contextuais (diversidade de assuntos e momentos de conversação, por exemplo) e escore total positivo. Por outro lado, as práticas negativas de educação, como bater e gritar, foram correlacionadas com problemas de comportamento das crianças e o escore total negativo avaliado pelo instrumento. Quanto às crianças, suas habilidades sociais estiveram positivamente relacionadas às variáveis contextuais e ao total positivo, enquanto problemas de comportamento relacionaramse ao escore total negativo.

Tabela 2 - Correlações entre o RE-HSE-P, a ECI A2 e o QRSH - Pais (Teste Não-Paramétrico de Spearman)

\begin{tabular}{|c|c|c|c|c|}
\hline \multirow[b]{2}{*}{$\begin{array}{c}\text { Categorias da ECI A2 e } \\
\text { QRSH - Pais }\end{array}$} & \multicolumn{3}{|c|}{ Categorias do RE-HSE-P } & \multirow[t]{2}{*}{ Total RE-HSE-P } \\
\hline & Comunicação & $\begin{array}{l}\text { Expressão de } \\
\text { sentimento e } \\
\text { enfrentamento }\end{array}$ & $\begin{array}{l}\text { Estabelecimento de } \\
\text { limites }\end{array}$ & \\
\hline \multicolumn{5}{|l|}{ ECI A2 } \\
\hline Respostas internalizantes & $-0,220$ & $-0,040$ & $-0,290$ & $-0,234$ \\
\hline Respostas externalizantes & $-0,097$ & 0,062 & $-0,436 * *$ & $-0,211$ \\
\hline Outras respostas & $-0,124$ & $-0,088$ & $-0,245$ & $-0,214$ \\
\hline Total & $-0,107$ & 0,032 & $-0,466^{* *}$ & $-0,259$ \\
\hline QRSH - Pais & & & & \\
\hline $\begin{array}{l}\text { Disponibilidade social e } \\
\text { cooperação }\end{array}$ & $0,388^{*}$ & 0,087 & $-0,004$ & 0,197 \\
\hline Interação social positiva & 0,150 & $0,382^{*}$ & $-0,119$ & 0,227 \\
\hline $\begin{array}{l}\text { Expressão de sentimento e } \\
\text { enfrentamento }\end{array}$ & $0,312^{*}$ & 0,295 & $0,387 *$ & $0,523 * *$ \\
\hline Total QRSH - Pais & $0,400^{* *}$ & $0,305^{*}$ & 0,188 & $0,455^{* *}$ \\
\hline
\end{tabular}

${ }^{*} \mathrm{p}<0,05 * * \mathrm{p}<0,01$

Conforme a Tabela 2, foram encontradas correlações significativas entre categorias do RE-HSEP e categorias da ECI A2 e do QRSH - Pais. Com relação à categoria comunicação, referente às HSE-P maternas, observa-se correlação positiva com as categorias disponibilidade social e cooperação, expressão de sentimentos e enfrentamento, e com o escore total no QRSH - Pais, sugerindo que as crianças cujas mães relataram apresentar habilidades de comunicação com maior frequência, apresentam mais respostas socialmente habilidosas. Resultado semelhante foi encontrado quanto à categoria expressão de sentimentos e enfrentamento, que também apresentou correlação positiva com respostas socialmente habilidosas das crianças envolvidas na categoria interação social positiva, e com o escore total do QRSH - Pais.

Já a categoria estabelecimento de limites apresentou correlação negativa com as repostas externalizantes e com o escore total da ECI A2, o que 
sugere que, quanto maior a frequência com que as mães relataram habilidades envolvidas no estabelecimento de limites, menor a frequência com que os filhos apresentavam problemas de comportamento externalizantes e de maneira geral. Por outro lado, essa categoria apresentou ainda correlação positiva com a categoria do QRSH - Pais, referente à expressão de sentimentos e enfrentamento.

Quanto ao escore total no RE-HSE-P, este apresentou correlação positiva com o escore total do
QRSH - Pais e, especificamente, com a categoria expressão de sentimento e enfretamento. Esses resultados sugerem que, quanto maior a frequência com que as mães relataram apresentar habilidades sociais educativas parentais, maior a frequência com que os filhos apresentavam respostas socialmente habilidosas, no seu ponto de vista. A Tabela 3 apresenta as comparações entre o grupo clínico e nãoclínico, de acordo com o CBCL, para as categorias do RE-HSE-P.

Tabela 3 - Média, desvio padrão e resultado do Teste Mann-Whitney (2-tailed) na comparação entre o grupo clínico e não-clínico para problemas de comportamento, conforme classificação do RE-HSE-P

\begin{tabular}{lccccc}
\hline \multirow{2}{*}{ Classificação do RE-HSE-P } & \multicolumn{3}{c}{ Clínico } & \multicolumn{3}{c}{ Não clínico } & \multicolumn{1}{c}{$p$} \\
\cline { 2 - 5 } & Média & DP & Média & DP & 0,028 \\
HSE-P & 8,14 & 2,27 & 11,25 & 3,11 & 0,005 \\
Negat & 12,29 & 4,19 & 5,83 & 1,95 & - \\
HS & 8,71 & 4,68 & 13,42 & 4,58 & 0,017 \\
Probl & 17,29 & 5,02 & 11,50 & 3,92 & - \\
Contexto & 15,14 & 6,01 & 14,58 & 7,79 & 0,017 \\
Total positivo & 8,29 & 2,29 & 12,08 & 3,48 & 0,001 \\
Total negativo & 33,29 & 8,01 & 20,08 & 5,63 & 0 \\
\hline
\end{tabular}

Nota. Clínico $-\mathrm{n}=7$; Não clínico $-\mathrm{n}=12$

Pela Tabela 3, notam-se diferenças estatisticamente significativas para a maioria dos itens avaliados pelo RE-HSE-P, de forma que o grupo nãoclínico apresenta mais habilidades sociais educativas e menos práticas negativas de educação. $\mathrm{Na}$ mesma direção, as crianças do grupo clínico emitem mais frequentemente comportamentos, problema, tais como desobediência, agressividade e descuido com o ambiente, do que as crianças do grupo não-clínico.

\section{Discussão}

De maneira geral, os resultados referentes às correlações observadas entre os diferentes instrumentos e as categorias comportamentais avaliadas reforçam a hipótese de que o comportamento dos filhos está relacionado às habilidades sociais educativas parentais. Em outras palavras, pode-se afirmar, que para esse grupo de mães separadas, os seus comportamentos influenciam os comportamentos dos filhos, explicitando contingências entrelaçadas (Glenn, 1986). Em diferentes análises e a partir de múltiplos instrumentos, constataram-se relações entre habilidades sociais educativas parentais, variáveis contextuais e habilidades sociais dos filhos. Por outro lado, práticas negativas de educação estiveram associadas a problemas de comportamento das crianças.

A análise das correlações mostrou que, enquanto a ocorrência de habilidades envolvidas na categoria comunicação e expressão de sentimento e enfrentamento estiveram positivamente associadas ao repertório socialmente habilidoso das crianças, o estabelecimento de limites esteve negativamente relacionado a respostas externalizantes e problemas de comportamento de maneira geral. Esses resultados remetem aos achados de outros estudos, como o de Bolsoni-Silva (2003), que constatou que habilidades para expressar sentimentos e habilidades envolvidas na comunicação eram mais relatadas por pais de crianças com indicativo de comportamentos socialmente habilidosos. Igualmente, no estudo de Salvo e cols. (2005) também foi observado que práticas envolvendo a demonstração de afeto estavam positivamente relacionadas à sociabilidade da criança.

No que diz respeito às habilidades envolvidas na categoria comunicação, situações de diálogo seriam especialmente importantes na medida em que possibilitam à mãe oferecer suporte ao filho, ajudandoo a lidar com dificuldades inerentes à transição na estrutura familiar (Wolchik \& cols., 2000). Em situações de conversação, as crianças poderiam encontrar nas mães uma fonte de apoio para compartilhar experiências sobre temas diversos, inclusive sobre seus sentimentos, principalmente no caso de famílias recém-separadas. Entretanto, salientam Wolchik e cols., quando nessas situações as mães abordam mais questões relacionadas à disciplina, isso diminui sua disponibilidade para tratar de temas de interesse para a criança, já que estariam mais atentas ao controle do seu comportamento do que a outros 
assuntos. Ainda assim, manter conversas sobre concepções de certo e errado também é importante para crianças nessa faixa etária, uma vez que na fase pré-escolar as crianças estão expostas a novos ambientes, além do familiar e, assim, precisam de orientação sobre regras de convívio social.

Quanto à relação entre a categoria que envolve a expressão de sentimentos e enfrentamento por parte das mães e a categoria interação social positiva, pode-se supor que o engajamento em situações que envolvem a demonstração de sentimentos, sejam eles positivos ou negativos, pode contribuir para a qualidade da interação. De acordo com Del Prette e Del Prette (2002b), a expressividade emocional favorece a harmonia entre as pessoas.

Segundo Hetherington e Stanley-Hagan (1999), é particularmente significante para uma criança que vive apenas com um dos pais a habilidade deste em oferecer afeto, ser comunicativo e atender às suas necessidades, considerando principalmente aquelas crianças cujos pais se separaram recentemente, pois o medo do abandono pela figura parental pode estar ainda presente. As ocasiões que envolvem a demonstração de sentimentos positivos e carinho podem servir para aproximar mãe e filho e para oferecer modelos de como demonstrar afeto, o que favoreceria a expansão das habilidades sociais das crianças. Além disso, nessas situações, as mães também podem oferecer importantes modelos para a expressão de sentimentos negativos, como descontentamento ante situações de desagrado. Para as crianças que ingressam na pré-escola, principalmente aquelas mais novas, encontrar alternativas socialmente habilidosas para lidar, por exemplo, com situações de desagrado diante de pares, pode ajudar a manter relações positivas e evitar a rejeição nesse contexto (Tremblay, Gervais \& Petitclerc, 2008). Isso, é claro, desde que as mães ofereçam modelos considerados socialmente habilidosos para expressar sentimentos negativos.

Favorecer, portanto, a expressão de sentimentos entre mães separadas e filhos pode contribuir para interações saudáveis da criança. Principalmente porque, de acordo com Pett e cols. (1999), mães separadas exercem mais controle negativo e mantêm mais trocas negativas com os filhos. Para Emery e Tuer (1993), isso ocorreria por causa da sobrecarga de atividades que podem contribuir para o uso de práticas coercitivas, sugerindo a necessidade de atenção à população de mães separadas a fim de auxiliar a ampliar seu repertório, aumentando as interações consideradas positivas.

A relação verificada entre o estabelecimento de limites e problemas de comportamento também tem sido sugerida pelos pesquisadores, como Tein e cols.
(2004), que observaram redução dos problemas de comportamento por meio da promoção de habilidades maternas envolvidas no estabelecimento de limites, como a consistência na disciplina. A consistência na disciplina pode ajudar a reduzir a frequência de interações coercitivas entre mães e filhos desencadeadas por questões sobre o "mau" comportamento da criança. Já a disciplina inconsistente poderia prejudicar a relação parental na medida em que diminuiria a disponibilidade da mãe como fonte de apoio e aumentaria as chances de usar práticas coercitivas (Wolchik \& cols., 2000).

Outro dado a ser destacado, a partir da Figura 1 e da Tabela 3, é a presença de crianças sem indicação clínica para problemas de comportamento e para habilidades sociais na amostra investigada, o que reforça a ideia de que nem todas as crianças apresentam problemas após a separação parental, desde que as interações sociais estabelecidas entre pais e filhos sejam satisfatórias (Hetherington \& StanleyHagan, 1999). Cabe lembrar, ainda, que a criança exerce um papel ativo no seu processo de socialização, interferindo também no relacionamento com seus cuidadores (Biasoli-Alves \& Sigolo, 1992). Ademais, assume-se que seu comportamento é multideterminado e, portanto, influenciado por outras variáveis, além das habilidades sociais educativas parentais. Por exemplo, em uma situação de separação conjugal, é importante levar em conta aspectos relacionados ao contato com o pai e à relação com o mesmo.

Considera-se que esse estudo pôde trazer contribuições para ampliar o conhecimento sobre habilidades sociais educativas de mães separadas e sua relação com o comportamento infantil. Os resultados do trabalho ajudam a reforçar a hipótese de que o comportamento dos filhos está relacionado às habilidades sociais educativas parentais e não poderiam ser atribuídos, portanto, apenas à separação, considerada uma variável meio-proximal.

Em um contexto de separação, a análise das relações entre o comportamento parental e o comportamento dos filhos merece especial atenção, pois as crianças apresentam desenvolvimento mais saudável em função das habilidades dos pais e não em virtude do seu status marital (Clarke-Stewart \& cols., 2000). Essa concepção favorece o entendimento sobre as variáveis que influenciam o comportamento infantil, como salientam Bolsoni-Silva e Marturano (2002), enfocando aspectos proximais do ambiente e não apenas características distais (Matos, 1983). Como limitação do estudo destaca-se, sobretudo, o tamanho da amostra que reduz possibilidades de generalização, especialmente na comparação entre população clínica e não-clínica, de acordo com o CBCL. 


\section{Referências}

Achenbach, T. M. \& Rescorla, L. A. (2001). Manual for ASEBA school-age forms \& profiles. Burlington, VT: University of Vermont, Research Center for Children, Youth, \& Families.

Almeida, C. G., Moraes, C. G. A., Perez, E A., Garcia, M. R. \& Pellizari, N. (2000). Pais separados e filhos: análise funcional das dificuldades de relacionamento. Estudos de Psicologia, 17(1), 31-43.

Alvarenga, P. \& Piccinini, C. (2001). Práticas educativas maternas e problemas de comportamento em préescolares. Psicologia: Reflexão e Crítica, 14(3), 449460.

Amato, P. R. (2000). The consequences of divorce for adults and children. Journal of Marriage and the Family, 62(4), 1269-1287.

Amato, P. R. \& Keith, B. (1991). Parental divorce and the well-being of children: a meta-analysis. Psychological Bulletin, 11(1), 26-46.

Biasoli-Alves, Z. M. M. \& Sigolo, S. R. R. (1992). Análise e categorização dos estudos sobre práticas de educação de crianças na família. Didática, 28, 103-114.

Bolsoni-Silva, A. T. (2003). Habilidades sociais educativas, variáveis contextuais e problemas de comportamento: comparando pais e mães de pré-escolares (Tese de Doutorado). Ribeirão Preto: Universidade de São Paulo.

Bolsoni-Silva, A. T. (2009). Estudos de confiabilidade e de validade do Roteiro de Entrevista de Habilidades Sociais Educativas Parentais (RE-HSE-P) (Tese de PósDoutorado). Ribeirão Preto: Universidade de São Paulo.

Bolsoni-Silva, A. T. \& Marturano, E. M. (2002). Práticas educativas e problemas de comportamento: uma análise à luz das habilidades sociais. Estudos de Psicologia, 7(2), 227-235.

Bolsoni-Silva, A. T., Marturano, E. M. \& Loureiro, S. R. (no prelo). Estudos de confiabilidade e validade do Questionário de Respostas Socialmente Habilidosas Versão para Pais - QRSH-Pais. Psicologia: Reflexão e Crítica.

Bolsoni-Silva, A. T., Marturano, E. M. \& Manfrinato, J. W. S. (2005). Mães avaliam comportamentos socialmente "desejados" e "indesejados" de préescolares. Psicologia em Estudo, 10(2), 245-252.

Bolsoni-Silva, A. T., Marturano, E. M., Figueiredo, V. A. P. \& Manfrinato, J. W. S. (2006). Habilidades sociais e problemas de comportamento de préescolares: comparando avaliações de mães e de professoras. Psicologia: Reflexão e Crítica, 19(3), 460469.

Bordin, I. A. S., Mari, J. J. \& Caeiro, M. F. (1995). Validação da versão brasileira do Child Behavior Checklist (CBCL) (Inventário de Comportamentos da Infância e Adolescência): dados preliminares. Revista da Associação Brasileira de Psiquiatria - Associação Psiquiátrica da América Latina, 17(2), 55-66.

Brenner, V. \& Fox, R. A. (1998). Parental discipline and behavior problems in young children. The Journal of Genetic Psychology, 159(2), 251-256.

Capaldi, D. M. \& Patterson, G. R. (1991). Relation of parental transitions to boys' adjustment problems: I. A linear hypothesis. II. Mothers at risk for transitions and unskilled parenting. Developmental Psychology, 27(3), 489-504.

Cherlin, A. J., Furstenberg, F. F., Chase-Lansdale, P. L., Kiernan, K. E., Robins, P. K., Morrison, D. R. \& Teitler, J. O. (1991). Longitudinal studies on effects of divorce on children in Great Britain and the United States. Science, 252(5011), 1386-1389.

Cia, F., Pereira, C. S., Del Prette, Z. A. P. \& Del Prette, A. (2006). Habilidades sociais parentais e o relacionamento entre pais e filhos. Psicologia em Estudo, 11(1), 73-81.

Clarke-Stewart, K. A., Vandell, D. L., McCartney, K., Owen, M. T. \& Booth, C. (2000). Effects of parental separation and divorce on very young children. Journal of Family Psychology, 14(2), 304-326.

Del Prette, A. \& Del Prette, Z. A. P. (2002a). Psicologia das relaçôes interpessoais: vivências para o trabalho em grupo. Petrópolis: Vozes.

Del Prette, A. \& Del Prette, Z. A. P. (2002b). Psicologia das habilidades sociais: terapia e educação. Petrópolis: Vozes.

Emery, R. E. \& Tuer, M. (1993). Parenting and the marital relationship. Em: T. Luster \& L. Okagali (Orgs.). Parenting: an ecological perspective (pp. 121 148). Hillsdate: Laurence Eilbaum.

Glenn, S. S. (1986). Metacontigencies in Walden Two. Behavior Analysis and Social Action, 5, 2-8.

Goldiamond, I. (2002). Toward a constructional approach to social problems: ethical and constitutional issue raised by applied behavior analysis. Behavior and Social Issues, 11(2), 108-197. (Texto original publicado em 1974). 
Gomide, P. I. C. (2001). Efeitos das práticas educativas no desenvolvimento do comportamento antisocial. Em M. L. Marinho \& V. E. Caballo (Orgs.). Psicologia Clínica e da Saúde (pp. 33-53). Londrina: Ed. UEL.

Graminha, S. S. V. (1994). A escala comportamental infantil de Rutter A2: estudos de adaptação e fidedignidade. Estudos de Psicologia, 11(3), 34-42.

Graminha, S. S. V. (1998). Recursos metodológicos para pesquisas sobre riscos e problemas emocionais e comportamentais na infância. Em G. Romanelli \& Z. M. M. Biasoli-Alves (Orgs.). Diálogos metodológicos sobre prática de pesquisa (pp. 7186). Ribeirão Preto: Legis Summa.

Hetherington, E. M. \& Stanley-Hagan, M. (1999). The adjustment of children with divorced parents: a risk and resiliency perspective. Journal of Child Psychology and Psychiatry, 40(1), 129-140.

Kaiser, A. P. \& Hester, P. P. (1997). Prevention of conduct disorder through early intervention: a social-communicative perspective. Behavioral Disorders, 22(3), 117-130.

Kerr, D. C., Lopez, N. L., Olson, S. L. \& Sameroff, A. J. (2004). Parental discipline and externalizing behavior problems in early childhood: the roles of moral regulation and child gender. Journal of Abnormal Child Psychology, 32(4), 369-383.

Lansford, J. E. (2009). Parental divorce and children's adjustment. Perspectives on Psychological Science, 4(2), 140-152.

Lansford, J. E., Malone, P. S., Castellino, D. R., Dodge, K. A., Pettit, G. S. \& Bates, J. E. (2006). Trajectories of internalizing, externalizing, and grades for children who have and have not experienced their parents' divorce or separation. Journal of Family Psychology, 20(2), 292-301.

Matos, M. A. (1983). A medida do ambiente de desenvolvimento infantil. Psicologia, 9(1), 5-18.

Morrison, D. R. \& Cherlin, A. J. (1995). The divorce and young children's well-being: a prospective analysis. Journal of Marriage and the Family, 57(3), 800-812.

Pacheco, J. T. B., Teixeira, M. A. P. \& Gomes, W. B. (1999). Estilos parentais e desenvolvimento de habilidades sociais na adolescência. Psicologia: Teoria e Pesquisa, 15(2), 117-126.
Patterson, G., Reid, J. \& Dishion, T. (2002). Antisocial boys: comportamento antisocial. Santo André: ESETec Editores Associados.

Pett, M. A., Wampold, B. E., Turner, C. W. \& Vaughan-Cole, B. (1999). Paths of influence of divorce on preschool children's psychosocial adjustment. Journal of Family Psychology, 13(2), 145164.

Pruett, M. K., Williams, T. Y. \& Insabella, G. (2003). Family and legal indicators of child adjustment to divorce among families with young children. Journal of Family Psychology, 17(2), 169-180.

Salvo, C. G., Silvares, E. F. M. \& Toni, P. M. (2005). Práticas educativas como forma de predição de problemas de comportamento e competência social. Estudos de Psicologia, 22(2), 187-195.

Tein, J. Y., Sandler, I. N., MacKinnon, D. P. \& Wolchik, S. (2004). How did it work? Who did it work for? Mediation in the context of a moderated prevention effect for children of divorce. Journal of Consulting and Clinical Psychology, 72(4), 617-624.

Tremblay, R. E., Gervais, J. \& Petitclerc, A. (2008). Prévenir la violence par l'apprentissage à la petite enfance. Montreal: Centre D'Excellence pour Le Développement dês Jeunes Enfants.

Whiteside, M. F. \& Becker, B. J. (2000). Parental factors and young child's post divorce adjustment: A meta-analysis with implications for parenting arrangements. Journal of Family Psychology, 14(1), 526.

Wolchik, S. A., Wilcox, K. L., Tein, J. Y. \& Sandler, I. N. (2000). Maternal acceptance and consistency of discipline as buffers of divorce stressors on children's psychological adjustment problems. Journal of Abnormal Child Psychology, 28(1), 87-102.

Wood, J. J., Repetti, R. L. \& Roesch, S. C. (2004). Divorce and children's adjustment problems at home and school: the role of depressive/withdrawn parenting. Child Psychiatry and Human Development, 35(2), 121-142.

Recebido em dezembro de 2009 Reformulado em abril de 2010 Aprovado em junbo de 2010 
Sobre as autoras:

Ana Carolina Villares Barral Villas Boas é mestre pelo Programa de Pós-Graduação em Psicologia do Desenvolvimento e Aprendizagem da Universidade Estadual Paulista - Bauru e especialista em Psicologia Clínica pelo HRAC/USP. Atualmente, cursa doutorado no Programa de Pós-Graduação em Processos de Desenvolvimento Humano e Saúde da Universidade de Brasília.

Alessandra Turini Bolsoni-Silva é professora do Departamento de Psicologia e da Pós-Graduação em Psicologia do Desenvolvimento e Aprendizagem da Universidade Estadual Paulista, Faculdade de Ciências, Bauru, São Paulo; doutora em Psicologia, pós-doutora em Medicina (USP-RP) e bolsista Produtividade CNPq.

Trabalho elaborado com base na dissertação de mestrado da primeira autora, sob orientação da segunda. Apoio financeiro: CAPES. 